\title{
Identifying Opportunities to Support Family Caregiving in Chile
}

\section{Francisco J. Gutierrez}

Department of Computer Science

University of Chile

Santiago, Chile

frgutier@dcc.uchile.cl

Sergio F. Ochoa

Department of Computer Science

University of Chile

Santiago, Chile

sochoa@dcc.uchile.cl

\section{Julita Vassileva}

Department of Computer Science

University of Saskatchewan

Saskatoon, SK, Canada

jiv@cs.usask.ca

Paste the appropriate copyright/license statement here. ACM now supports three different publication options:

- ACM copyright: ACM holds the copyright on the work. This is the - historical approach.

- License: The author(s) retain copyright, but ACM receives an

- License: The author(s) retain

- Open Access: The author(s) wish to pay for the work to be open

- Open Access: The author(s) wish to pay for the wor
access. The additional fee must be paid to ACM.

This text field is large enough to hold the appropriate release statement assuming it is single-spaced in Verdana 7 point font. Please do not change the size of this text box.

Each submission will be assigned a unique DOI string to be included here.

\begin{abstract}
Once older adults become less self-sufficient, one or more adult children often assume the role of family caregiver. Unfortunately, this situation is usually perceived as stressful by caregivers. To better

understand this process, we interviewed

intergenerational triads: older adults, their adult

children acting as caregivers, and their grandchildren.

The study results show that the commitment of

caregivers is quite strong regardless of the place where they live. In addition, the gender of older adults and caregivers affects the dynamics and complexity of the process. Through this study we aim to identify

opportunities to support family caregiving, respecting the preexisting relations, attitudes, needs, and expectations of the involved stakeholders.
\end{abstract}

\section{Author Keywords}

Family caregiver; family communication; older adults

\section{ACM Classification Keywords}

H.5.3. Group and Organization Interfaces: Computersupported cooperative work.

\section{Introduction}

The world population is dramatically getting older due to increased life expectancy and dropping birth rates. Frequently, when the elderly become less self- 
sufficient, their families assume a caregiver role. This role typically falls on one or more adult children, who engage in providing emotional support and helping the elderly maintain a reasonable quality of life.

While the adult children accepting these duties usually do so in response to the affection and protection they received during childhood [3], cultural factors largely influence the extent to which these people get involved in the process. For instance, Latino family members typically tend to perceive this obligation stronger than people raised in America or Western Europe [11].

Although family caregivers sometimes include their partner and their children in the caring process, they usually struggle with maintaining their own family as well as providing care to their parents [5].

Unfortunately, this situation is not free from tensions that frequently burden the caregiver $[5,12]$.

Aiming to identify opportunities to design computersupported solutions for assisting family caregivers, we explored how this process is perceived and conducted in middle class Chilean families living in urban areas. By interviewing intergenerational triads from the same family branch, we identified asymmetric perceptions on how the caregiving duties are fulfilled, as well as how family caregivers communicate and coordinate their actions with other relatives. Building on these findings, we identified family caregiving scenarios showing opportunities to design computer-supported technology for helping adult children fulfill their duties.

\section{Related Work}

The design of effective computer-supported solutions to assist people in caregiving settings has to consider the needs of both care recipients and caregivers [1]. While a broad amount of research in this topic has been conducted in medical scenarios (e.g., $[8,9]$ ), there are open challenges concerning the design and usage of technology for supporting such a process in family settings.

Even though family relations are complex, and are sustained in deep affection among its members, they are not free from tensions. For instance, one of the most significant sources of conflict between older adults and adult children is related to the management of communication with each other [2]. These conflicts usually correspond to misaligned perceptions on how older adults communicate with their adult children.

Distance is also a typical hurdle that family members need to overcome for maintaining periodic interaction and a meaningful sense of connection. Forghani and Neustaedter [4], studying the communication patterns of distance-separated grandparents and grandchildren, identified that adult children usually assume the role of family communication broker in intergenerational exchanges, thus providing the means to share information between people that do not have frequent contact. Similarly, Gutierrez and Ochoa [5] identified that adult children -and sometimes grandmothers- also assume a similar role aiming to provide indirect

communication means as a way to sustain the affective link among loosely coupled people.

Although several computer-supported systems have been proposed to facilitate interactions between family members and older adults, the adoption of these technologies by the latter is usually complex. Huber et al. [6] identified that one of the most important factors 


\begin{tabular}{ll|rrr} 
& & OA & AC & GC \\
\hline Male & N & 4 & 5 & 9 \\
& Age & 78 & 50 & 23 \\
Female & $\mathrm{N}$ & 12 & 11 & 7 \\
& Age & 70 & 47 & 21
\end{tabular}

Table 1: Gender and mean age of the interviewed people. OA stands for Older Adults, AC for Adult Children, and GC for

Grandchildren. that limits the adoption of technology by older adults is the fear that these systems might replace human contact with their family caregivers. Therefore, one plausible alternative to assist this group of people is to design meaningful experiences that do not limit physical exchanges with their caregivers. Respecting the existing routines and expectations of the different involved stakeholders is required not only for facilitating the technology adoption and promoting effective and meaningful exchanges, but also to try aligning the implicit and explicit asymmetries existing across generations [7, 10].

This work builds on previous research by exploring how family caregivers get involved and manage their exchanges with their elders and other family members. Our study shows that family caregivers are concerned about coordination and negotiation issues, as well as any passive attitudes of other family members.

\section{Study Methodology}

Our study was based on interviews exploring the communication and coordination practices of adult children assuming the role of family caregiver.

\section{Participants}

Sixteen intergenerational triads were recruited through snowball sampling in the metropolitan area of Santiago, Chile. These triads are composed of an older adult, one of the adult children acting as his/her family caregiver, and one of the grandchildren from the same family branch. Given that the literature on family caregiving reports that women tend to assume this role in a higher number than men [12], we stratified our sample of adult children to reflect this in our study. Table 1 shows the socio-demographic features of the participants.

\section{Interview method}

We conducted individual semi-structured interviews in the participants' home, lasting between 45 and 60 minutes each. Interview scripts were composed of two main sections: a common one for all participants aiming to explore the general interaction routines and perceived affinity structure of the family links, and a second one differentiated for each generation in the triad. Sample questions from the latter section were: Do you think that your children have the obligation of caring for you? Why? (older adults); What challenges do you face when caring for your parents? (adult children); and Would you rather let your parents take care of your grandparents without involving you? Why? (grandchildren). The interview protocol was piloted with a sample of three families, in order to limit wording problems and ambiguous statements.

Data collection and analysis

Interviews were conducted in Spanish and were audiorecorded with the consent of each participant. The audio data was later transcribed and extended with handwritten field notes taken during each interview. We performed open, axial, and selective coding aiming to understand the emerging communication and coordination practices, as well as issues that family caregivers need to overcome when fulfilling their duties. Similarly, we analyzed how these caregivers position themselves within their families and what their expectations regarding other family members are.

\section{Results}

The filial obligation shapes the relationship between adult children and their parents, and the parenting style fits the interactions between adult children and their own children. The relationship between grandchildren 

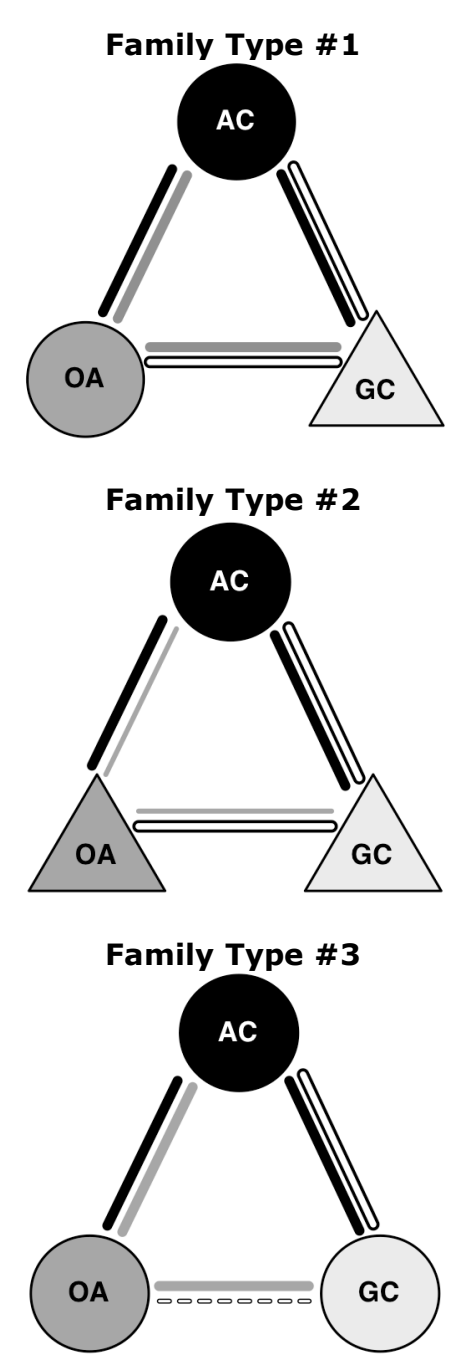

Figure 1: Representative intergenerational triads. and grandparents seems to be ruled by the number and quality of the shared memories among them, which in turn depends on the physical distance between these people. Through our interviews, we corroborated previous research, which indicates that distance usually does not deteriorate the link between an adult child acting as family caregiver and his/her parents. This is due to the underlying filial obligation mediating this relation. However, it does negatively impact the grandparent-grandchild relationship [5].

In an initial attempt to describe the communication and coordination practices of caregivers across different family structures, we grouped the interviewed triads under one of the three family types shown in Figure 1. We used circles to depict women and triangles for men. The quality of the affective link is represented with a line between the involved people. The thickness of this link maps the perceived strength of the relationship between each member of the triad. The color of the line indicates who evaluated the quality of each link.

Family Type \#1 (FT1) groups families 3, 4, 7, 9, 11 12 , and 14 , where one daughter usually acts as caregiver and the care recipient is a female older adult. This family type, which is the most frequent one in our sample, sometimes involves one or more grandchildren in the caregiving process. Family Type \#2 (FT2) comprises families $6,10,15$, and 16 , and it involves male and female caregivers. In this case, the care recipient is a male older adult. Finally, Family Type \#3 (FT3), comprises families 1, 2, 5, and 13, and represents triads where the adult children and grandchildren live far away from the older adult; e.g., in a different city. This involves male and female caregivers, and the care recipients are mainly female older adults. Naturally, given the complexity of family links, not all families fit under the identified groups. This is particularly the case of family 8 , which was analyzed individually. The next subsections outline the main findings of our study.

The affective link is usually strong and symmetric between a caregiver and her mother and children (FT1 and FT3), and asymmetric with the father (FT2)

The interviews show that family caregivers are highly engaged in caring for their parents; quoting AC3

(family 3, female, 54 years old): "My mom needs me to care for her. She needs affection, that we go visit her, or at least that we spend some time together watching $T V^{\prime \prime}$. As another example, AC7 (male, 39 years old) takes the initiative in coordinating the family efforts toward their elderly as a way to fulfill the underlying duties of filial obligation: "I am always paying attention to what we can do as a family to be closer. [...] As the eldest one among my siblings, I feel I have the responsibility of being in charge of the other members in my family". The caregiving process is usually accepted and highly appreciated by their parents particularly the mother- and children. For that reason, the relationship among these people tends to be strong and symmetric.

Contrarily, the interviewed grandfathers tend to refuse the support provided by family caregivers. For instance, OA6 (male, 75 years old) said: "My children are all grown up now. They need to take care of their own families. This is my time and I want to spend it on my own". These people also consider that adult children and grandchildren frequently invade their privacy and restrict their independence, which may explain the asymmetry in the perceived affective link shown in FT2. 

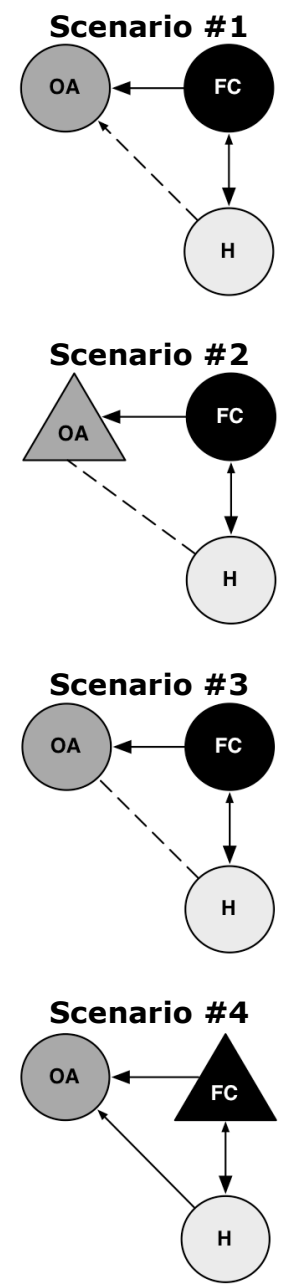

Figure 2: Scenarios of family caregiving support. OA stands for Older Adult, FC for Family Caregiver, and $\mathrm{H}$ for Helper.
Female caregivers expect more involvement from other family members

Our interviews also show that older adults usually have two or three family caregivers, who also take care of their own children while trying to find an appropriate work-life balance. As a result, this situation generates care duties being assumed in an asymmetric way by family caregivers, where their own children tend to have priority. Therefore, the tasks derived from caring for their parents are frequently perceived as a burden that they have to assume due to their filial obligation. This produces periods of high stress in family caregivers that are usually better addressed by men. Male caregivers tend to show having more control of the coordination process and find the means to ask for help and involve other people, particularly family members of their same branch. For example, AC9 (male, 43 years old) relies on his brothers when he is unavailable: "I always try to support my parents. My brothers also care for them, but at a lesser extent. [...] When you ask them to do something, they are there. We are very united, so we share responsibilities".

Contrarily, in the case of female caregivers, our interviews repeatedly show that they lack the means to ease this coordination process. Therefore, they have to address their challenges by themselves, leading to complaints about the lack of help, particularly toward family members of their same generation. For instance, AC10 (female, 42 years old) said: "Dad is already used to me being the one who drives him to the doctor. [...] I have to do it because I live closer (to him). [...] I would really like more involvement from my brothers.

Although they are aware that they need to do more for our dad, they tend to overload me and avoid their responsibilities".
All in all, it turns out that female caregivers seem to expect more involvement from other family members, whereas male caregivers actually recognize that they benefit from more support, particularly that offered by members of their same generation. These observations resonate with related research, particularly in the case of burdened family caregivers coping with older relatives suffering from dementia [12].

\section{Discussion}

Considering the study results we identified four caregiving scenarios (Fig. 2), which can be supported in different ways using technology. Scenario \#1 is the most frequent one, where a female family caregiver simultaneously takes care of her mother and own children. The size of the arrows indicates the willingness of a person to support the other party, and the type of line maps if the support is effective (solid lines) or potential (dashed lines)

Regarding scenario \#1, our study indicates that grandchildren and some adult children are not available to be caregivers; however, they are willing to contribute as much as possible -a role that we identify as helpers-. Unfortunately, they do not frequently assume such a role since they have little to no understanding of the elderly short-term needs; e.g., buying medicine or groceries. Female older adults receiving help usually do not represent an obstacle for helpers. This opens several opportunities for designing technology to engage and support helpers, thus satisfying the needs of older adults and reducing the workload of caregivers. Nevertheless, designing this kind of technology requires that female caregivers realize that other family members can contribute not only as caregivers, but also as helpers. In this sense, 
designing technology that decentralizes (even partially) the control held by female caregivers represents a plausible design opportunity.

Scenario \#2 is similar to the previous one, with the difference that the older adult is a man. In this case, there is not only a lack of visibility of the elderly shortterm needs, but also a reluctance of older adults to receive external support. Therefore, effective technology to address the caregiving process should probably be aimed at persuading both helpers and older adults using positive computing solutions. For instance, highlighting shared memories can contribute to increasing the willingness of potential helpers to assist the elderly, and to the latter for receiving external support. There is also an opportunity to assist male older adults using their partner as mediator; i.e., the grandmother can make their shared needs explicit and thus getting external support that benefits both of them.

In scenario \# 3 the caregiver and her children live far away from the older adult. Typically, the older adults keep direct contact with their caregiver, but usually not as much with their grandchildren. Although there is technology specifically targeted at keeping alive and reinforcing the link between distance-separated grandparents and grandchildren, most of these solutions are focused on kids and do not necessarily consider teen or young adult grandchildren as potential actors in the caregiving process. This opens several opportunities to design technology for this scenario.

Finally, in scenario \#4 the caregivers are male.

Although they usually involve other family members as helpers, their coordination is centralized. Therefore, it depends on the active participation of caregivers. Coordinating activities in this scenario can benefit from technology that decentralizes or automates part of this process, given that male caregivers usually receive external support from other family members.

\section{Conclusions and Future Work}

The process of caring for older adults in Chile usually burdens family caregivers, who have to take care of their own children and their parents while trying to reach an appropriate work-life balance. Through an intergenerational interview study, we identified several caregiving scenarios and opportunities to support them using technology. Although a family ecosystem can involve combinations of these proposed scenarios, thus increasing the complexity of designing effective mechanisms to ease family caregiving, this research shows initial steps on how to deal with this problem.

Given the small number of participants and the cultural aspects involved in our study sample, the findings and proposed design opportunities may not be applicable to other scenarios. In this sense, our future work will focus on building upon our initial findings by interviewing and analyzing additional intergenerational triads, as well as prototyping and evaluating in the field the effectiveness and acceptance of design alternatives.

\section{Acknowledgments}

This work has been partially supported by the Fondecyt Project (Chile), grant: 1150252. The work of Francisco J. Gutierrez has been supported by the Ph.D.

Scholarship Program of Conicyt Chile (CONICYTPCHA/Doctorado Nacional/2013-21130075). 


\section{References}

1. Yunan Chen, Victor Ngo, and Sun Young Park. 2013. Caring for caregivers: designing for integrality. In Proceedings of the ACM Conference on Computer-Supported Cooperative Work (CSCW'13), 91-102.

http://dx.doi.org/10.1145/2441776.2441789

2. Edward J. Clarke, Mar Preston, Jo Raskin, and Vern L. Bengtson. 1999. Types of conflicts and tensions between older parents and adult children. The Gerontologist 39, 3, 261-270.

http://dx.doi.org/10.1093/geront/39.3.261

3. Melanie M. Domenech Rodríguez, Melissa R. Donovic, and Susan L. Crowley. 2009. Parenting styles in a cultural context: observations of "protective parenting" in first-generation Latinos. Family Process 48, 2, 195-210.

4. Azadeh Forghani and Carman Neustaedter. 2014 The routines and needs of grandparents and parents for grandparent-grandchild conversations over distance. In Proceedings of the SIGCHI Conference on Human Factors in Computing Systems (CHI'14), 4177-4186. http://dx.doi.org/10.1145/2556288.2557255

5. Francisco J. Gutierrez and Sergio F. Ochoa. 2016. Mom, I do have a family!: attitudes, agreements, and expectations on the interaction with Chilean older adults. In Proceedings of the ACM Conference on Computer-Supported Cooperative Work and Social Computing (CSCW'16). http://dx.doi.org/10.1145/2818048.2820000

6. Lesa Huber, Kalpana Shankar, Kay Connelly, Kelly E. Caine, L. Jean Camp, Beth Ann Walker, and Lisa Borrero. 2013. How in-home technologies mediate caregiving relationships in later life. International Journal of Human-Computer Interaction 29, 7, 441455.

http://dx.doi.org/10.1080/10447318.2012.715990
7. Siân E. Lindley, Richard Harper, and Abigail Sellen. 2008. Designing for elders: exploring the complexity of relationships in later life. In Proceedings of the BCS Conference on HumanComputer Interaction ( $\left.\mathrm{HCI}^{\prime} 08\right)$, 77-86.

8. Leslie S. Liu, Sen H. Hirano, Monica Tentori, Karen G. Cheng, Sheba George, Sun Young Park, and Gillian R. Hayes. 2011. Improving communication and social support for caregivers of high-risk infants through mobile technologies. In Proceedings of the ACM Conference on Computer-Supported Cooperative Work (CSCW'11), 475-484. http://dx.doi.org/10.1145/1958824.1958897

9. Andrew D. Miller, Sonali R. Mishra, Logan Kendall, Shefali Haldar, Ari H. Pollack, and Wanda Pratt. 2016. Partners in care: design considerations for caregivers and patients during a hospital stay. In Proceedings of the ACM Conference on ComputerSupported Cooperative Work and Social Computing (CSCW'16).

http://dx.doi.org/10.1145/2818048.2819983

10. Karyn Moffatt. 2013. Older-adult HCI: why should we care? Interactions 20, 4 (July + August 2013), 72-75.

http://dx.doi.org/10.1145/2486227.2486242

11. National Alliance for Caregiving and AARP. 2015. Caregiving in the United States - 2015. Retrieved January 7, 2016 from

http://www.aarp.org/ppi/info-2015/caregiving-inthe-united-states-2015.html

12. Evridiki Papastavrou, Athena Kalokerinou, Savvas S. Papacostas, Haritini Tsangari, and Panagiota Sourtzi. 2007. Caring for a relative with dementia: family caregiver burden. Journal of Advanced Nursing 58, 5 (June 2007), 446-457. http://dx.doi.org/10.1111/j.13652648.2007.04250.x 